\title{
COORDINACIÓN MOTRIZ E ÍNDICE DE MASA CORPORAL EN ESCOLARES DE SEIS CIUDADES COLOMBIANAS
}

\section{MOTOR COORDINATION AND BODY MASS INDEX OF PUPILS OF SIX COLOMBIAN SCHOOLS}

\author{
José Armando Vidarte-Claros ${ }^{1 *}$, Consuelo Vélez Álvarez², José Hernán Parra-Sánchez ${ }^{3}$
}

\begin{abstract}
${ }^{1}$ Profesor-investigador, Departamento de Movimiento Humano, Facultad de Salud, Líder del Grupo de investigación Cuerpo-Movimiento. Universidad Autónoma de Manizales, Manizales-Caldas, Colombia, e-mail: Jovida@autonoma.edu.co; ${ }^{2}$ Profesora-investigadora, Departamento de Salud Pública, Facultad de Salud, Grupo de investigación Cuerpo-Movimiento. Universidad Autónoma de Manizales, Manizales-Caldas, Colombia, e-mail: cva@autonoma.edu.co; ${ }^{3}$ Profesor-investigador, Departamento de Economía. Universidad Nacional de Colombia, sede Manizales, e-mail: jhparrasa@unal.edu.co; *Autor para correspondencia
\end{abstract}

\author{
Rev. U.D.C.A Act. \& div. Cient. 21(1): 15-22, Enero-Junio, 2018 \\ https://doi.org/10.31910/rudca.v21.n1.2018.658
}

\section{RESUMEN}

Un déficit madurativo de la coordinación, respecto a los niveles correspondientes con la edad cronológica, puede conllevar, en el niño, una serie de trastornos en el desarrollo de las capacidades coordinativas, cuando ejecuta actividades motrices. El objetivo del presente estudio fue correlacionar la coordinación motriz con el Índice de Masa corporal (IMC), en escolares entre 10 a 12 años, de seis ciudades de Colombia. Fue un estudio descriptivo, con fase correlacional, donde participaron 2.651 niños. La medición de la coordinación motriz fue realizada con el test KTK (Körperkoordinations Test für Kinder), previo diligenciamiento del asentimiento y del consentimiento informado. Los resultados muestran que la relación de participantes entre hombre y mujer fue de 1:1, la media de la edad fue de 10,9 $\pm 0,81$ años. Además, se encontró que existen diferencias estadísticamente significativas entre la coordinación motriz, el IMC y las diferentes variables de estudio y correlaciones significativas e inversas, entre la coordinación motriz y el IMC, en todos los grupos de las variables estudiadas.

Palabras clave: Obesidad, rendimiento psicomotor, actividad física, desempeño motor, antropometría.

\section{SUMMARY}

A maturational deficit of coordination, with respect to the levels corresponding to chronological age, can lead to a series of disorders in the development of coordinative capacities in the child, when executing motor activities. The objective of the present study was to correlate motor coordination with the Body Mass Index (BMI), in schoolchildren between 10 and 12 years of age in six cities of Colombia. It was a descriptive study with a correlation phase, involving 2651 children, the measurement of motor coordination was performed with the KTK test (Körperkoordinations Test für Kinder), after completion of the consent and informed consent. The results show that the ratio of participants between men and women was 1 : 1 , the average age was 10,9 $\pm 0,81$ years, and it was found that there are statistically significant differences between motor coordination, BMI and the different study variables and significant and inverse correlations between motor coordination and BMI in all groups of the variables studied.

Key words: Obesity, psychomotor performance, physical activity, motor performance, anthropometry.

\section{INTRODUCCIÓN}

La coordinación motriz, se puede definir, como "la capacidad de ordenar y organizar las acciones motrices orientadas hacia un objetivo determinado con precisión, eficacia, economía y armonía, lo que requiere la actividad del sistema nervioso que integra todos los factores motores sensitivos y sensoriales necesarios para la realización adecuada de movimientos" (Hernández et al. 2004; Muñoz-Rivera, 2009) y ha sido asumida como el mayor nivel de complejidad de una tarea motriz, ya que exige altos niveles de complejidad, necesarios para el desempeño eficaz (Gallahue \& Ozmun, 2005).

Ruiz-Pérez (2005) establece que un déficit madurativo de la coordinación respecto a los niveles correspondientes con la edad cronológica, origina deficiencias en el desarrollo de las capacidades coordinativas y una serie de trastornos, como asimetrías en las acciones corporales; problemas de equilibrio dinámico, inestabilidad y temor; inestabilidad y falta de control motor tras realizar tareas complejas; incapacidad 
para seguir ritmos; incapacidad para controlar la fuerza y dificultades en la planificación motriz de las acciones, entre otros.

Algunas de las limitaciones que pueden afectar la coordinación motriz son, entre otros, el nivel de actividad física; la edad; la fatiga, tanto física como psíquica; el nivel de aprendizaje; el segmento corporal implicado; la simetría de movimientos y el sentido de dirección del movimiento (Hernández et al. 2004; Gallahue \& Ozmun, 2005).

Actualmente, los estilos de vida que mantienen los niños no son activos y se convierten en factores predisponentes al sobrepeso (disminución de tiempo dedicado a la actividad física, disminución en la capacidad aeróbica y el aumento de tiempo dedicado a actividades sedentarias, como ver televisión, jugar videojuegos y la Internet) (OPS/OMS, 2003; Fernández, 2005; Ramos, 2006; Duque \& Parra, 2012).

Por tanto, la actividad física desempeña un papel importante en la prevención de enfermedades crónicas no trasmisibles y disminuye el riego de obesidad, actuado en la regulación del balance energético y preservando o manteniendo la masa magra en detrimento de la masa grasa (Secien-Palacin \& Jacoby, 2003).

Diferentes estudios muestran cómo el desarrollo de la coordinación motriz en la infancia puede influir decisivamente en la motivación e implicación -de manera positiva-, con el logro de la actividad física, la participación deportiva y la posibilidad de obtener adecuados rendimientos académicos (Graham et al. 2011; Lopes et al. 2013); los buenos niveles de coordinación motriz pueden estar asociados a valores mas elevados de actividad física y ser un factor preventivo del desarrollo de exceso de peso (Lopes et al. 2012; Lopes et al. 2011; Robinson et al. 2015). Los niños con niveles bajos de coordinación motriz tienen un mayor riesgo de tener sobrepeso, obesidad y este riesgo aumenta con la edad (Lopes et al. 2014).

Conocer los niveles de coordinación motriz de la población escolar ha dado lugar a que se adopten protocolos y criterios de evaluación y valores de corte, establecidos para la población de países desarrollados, que presentan realidades diferentes a las del contexto nacional. Dichos procesos buscan brindar herramientas claves, para orientar los diferentes procesos de intervención, dirigidos a esta población, posibilitando, finalmente, mejores estrategias de desarrollo, a nivel individual, colectivo y en la esfera biopsicosocial de los niños, que posibiliten un mejor y adecuado rendimiento motriz y académico.

El objetivo fue correlacionar la coordinación motriz con el IMC, en escolares entre 10 a 12 años, acorde a lo estipula- do, donde la coordinación, como capacidad motriz, ya se encuentra establecida y se evidencia de manera motriz y biológica en estas edades. Las ciudades participantes fueron establecidas por la posibilidad de acceder a la recolección de información, ya que fue un trabajo desarrollado desde un macroproyecto en la maestría de la Universidad Autónoma de Manizales.

\section{MATERIALES Y MÉTODOS}

Tipo de estudio: Estudio descriptivo, con fase correlacional, realizado con niños entre 10 y 12 años de edad, escolarizados en instituciones públicas y privadas de las ciudades de Manizales (Caldas), Riohacha (Guajira), Popayán (Cauca), Guadalajara de Buga, (Valle del Cauca), Yarumal (Antioquia) y Zarzal (Valle del Cauca) (Colombia). Se empleó un muestreo aleatorio estratificado, con afijación proporcional, con un margen de error usado de $0,3 \mathrm{~kg} / \mathrm{m}^{2}$ y una confiabilidad del $95 \%$

Participantes: El total de la muestra fue de 2.651 niños escolares, distribuidos así: Manizales, 448; Popayán, 442; Riohacha, 441; Yarumal, 440; Buga, 440 y Zarzal, 440 niños. Para la estimación de la varianza, se tomó como base, el estudio "Exposición a pantallas, sobrepeso y desacondicionamiento físico en niños y niñas" (Duque \& Parra, 2012). Fueron criterios de inclusión, el estar matriculado en la institución educativa, tener la edad entre 10 y 12 años, participar voluntariamente y haber diligenciado el consentimiento informado, por parte del padre o acudiente. Este trabajo fue aprobado por el comité de Bioética, de la Universidad Autónoma de Manizales, según acta No. Acta 039, del 8 de octubre de 2014.

Procedimiento: Los materiales utilizados en el desarrollo de las diferentes pruebas fueron los siguientes:

En la toma del peso, se usó una báscula marca Tanita BC 543 precisión de $1 \mathrm{~g}$ y para la toma de la talla, el estadiómetro móvil seca 217 , precisión de $1 \mathrm{~mm}$, los cuales, fueron calibrados cada 100 evaluaciones, tal como lo refiere la casa distribuidora.

Los materiales usados en la prueba KTK fueron elaborados, según las especificaciones establecidas en el protocolo del test de coordinación. La prueba KTK ha sido diseñada para identificar y para diagnosticar a niños con dificultades de movimiento y de coordinación, entre los 5 y los 14 años. Las pruebas pueden ser aplicadas de forma individual, con Fiabilidad 0,65 a la 0,87 (Kiphard \& Schilling, 1974; Vandorpe \& Schilling, 2011; Vidal et al. 2009; Valdivia et al. 2008) y su aplicación tiene una duración aproximada 10 a 15 minutos, por niño.

La prueba consiste en cuatro tareas, las cuales, siempre son demostradas inicialmente por el evaluador y el niño puede 
hacer hasta dos ensayos, según la tarea: la primera tarea es la barra de equilibrio, que consiste en mantener el equilibrio mientras se camina hacia atrás, en 3 listones de madera, de $3 \mathrm{~m}$ de largo, $3 \mathrm{~cm}$ de alto y anchuras de $3 \mathrm{~cm}$, 4,5cm y $6 \mathrm{~cm}$; en cada uno de los tres listones hay tres realizaciones válidas. Una vez realizado el ensayo, el niño intenta pasar el listón caminando solo hacia atrás y tres veces por listón. Si durante un intento se cae, se contabilizan los pasos (un paso equivale a un punto) y se pasa al siguiente intento; pasar el listón sin caerse son 8 puntos. A continuación, repetirá la operación en el listón de $4,5 \mathrm{~cm}$ y en el de $3 \mathrm{~cm}$.

La segunda tarea son los saltos unipodal, que consiste en saltar con una pierna por encima de planchas de gomaespuma, apiladas unas encima de otras. Se sale con la pierna de salto en apoyo y la otra flexionada atrás, desde detrás de una línea situada a 1,50m del obstáculo. El primer salto es de aproximación al obstáculo, el segundo es para superarlo $y$, luego, hay que hacer dos saltos más (botes con una pierna), para demostrar que el salto es controlado y se mantiene el equilibrio. Las alturas iniciales, se determinan con los resultados de estos ensayos y la edad de los participantes; se deben superar hasta alcanzar las 12 espumas apiladas, que miden $50 \times 20 \times 5 \mathrm{~cm}$ de altura.

La tercera tarea son saltos laterales, que se hacen sobre dos planchas de contrachapado de 60 × 50 x 0,8cm de grueso, atornilladas juntas y preparadas a prueba de deslizamientos. En su parte central va atornillada una tira de madera de 60 x 4 x 2cm de alto; además se requiere de un cronómetro. La tarea consiste en saltar lateralmente a uno y otro lado de la tira de madera, tan rápido como sea posible, durante 15 segundos. Los pies deben despegar de un lado de la tira y aterrizar en el otro, de forma simultánea.

La última tarea son los desplazamientos laterales, que consiste en desplazar tantas veces como sea posible, en 20 segundos, dos tablas lateralmente de 25 x 25 x 1,5cm de grueso. El niño sube a una tabla dejando la otra a su izquierda; a continuación, toma la tabla de su izquierda con las dos manos y la pone a su derecha; después, se sube encima de ésta y de nuevo toma la tabla de la izquierda y así, sucesivamente. Toda la prueba reclama un espacio tranquilo, alejado de distracciones, con unas dimensiones mínimas de 4 x 5m, en el que el sujeto realiza las pruebas, de forma individual.

La coordinación total fue clasificada en los siguientes niveles: muy malo, malo, regular, bueno y muy bueno, por cada uno de los rangos de edad del niño, los cuales, son producto de la sumatoria de los valores en cada una de las pruebas establecidas, tal como se planteó en la introducción. En el presente estudio, se abordaron las edades entre 10 y 12 años, como aspectos que indican la consolidación de la coordinación en los niños.
Análisis estadístico: Los datos fueron procesados y analizados en el programa SPSS Versión 21.0, licenciado por la Universidad Autónoma de Manizales. Se realizó un análisis univariado, que posibilitó la presentación de los datos, mediante tablas de frecuencias absolutas y relativas. Se calcularon medidas de tendencia central y de variabilidad o dispersión para las variables. Inicialmente, se realizó el test de normalidad (Kolmogorov-Smirnov), de la variable coordinación, para cada uno de los grupos de las variables (ciudad, tipo colegio, sexo y edad). Para el análisis bivariado, se buscó establecer la asociación entre las variables de estudio. Para determinar la relación lineal entre las variables, se utilizó el coeficiente de correlación de Pearson y la significancia estadística, se estableció cuando los valores de $\mathrm{p}<0,05$.

\section{RESULTADOS Y DISCUSIÓN}

Participaron en el estudio 2.651 niños escolares, entre 10 y 12 años de edad; la relación hombre - mujer fue de 1:1; el $36,1 \%$ correspondió a niños de 10 años; 32,7\%, de 11 años y 31,2\% tenían 12 años; la media de la edad fue de 10,9 años + 0,81 años. En mayor porcentaje, los niños cursaban los grados 5 primaria y 6 bachillerato, en colegios oficiales.

El resultado de este test muestra que la variable coordinación motriz no presenta distribución normal en ninguno de los grupos de las variables mencionadas; por lo anterior, se efectúa el test no paramétrico de Kruskal Wallis, en el caso de la comparación de promedios de la ciudad y de la edad y el test de U Mann Whitney, para el tipo de colegio y el sexo.

Como lo muestra la tabla 1 , se presentan diferencias estadísticamente significativas en el promedio de la coordinación motriz en las seis ciudades, siendo significativamente superior este promedio, para Manizales y Buga; para el tipo de colegio no se evidencian diferencias significativas. Por sexo, los hombres presentan una mejor coordinación que las mujeres, siendo estas diferencias, estadísticamente significativas. Por último, a mayor edad mejores niveles de coordinación y estas diferencias fueron estadísticamente significativas $\mathrm{p}<0,05$.

Al comparar las medias del IMC por sexo y por edad, se observa que, en los niños de 11 y 12 años, las mujeres presentan una media de IMC mayor que los hombres, siendo esta diferencia estadísticamente significativa $(p<0,05)$, en los niños de 12 años (Tabla 2).

Se presentan diferencias estadísticamente significativas en el promedio del índice de masa corporal en las seis ciudades, siendo significativamente superior este promedio, para Zarzal, Popayán y Buga; para el tipo de colegio, se evidencian diferencias significativas. Por sexo, los hombres presentan un mejor IMC que las mujeres, siendo estas diferencias, estadísticamente significativas. Por último, a mayor edad más 
altos los niveles de IMC y estas diferencias son significativas (Tabla 3).

Se evidencia, en el presente estudio, cómo al comparar el IMC por edad y por sexo las niñas de 11 y 12 años son quienes presentan una media de IMC mayor y esta diferencia es estadísticamente significativa, en los de 12 años $(p<0,05)$. Al comparar estos datos, se encontró cómo Chivers et al. (2013) muestran, en su estudio realizado en niños de 10 a 14 años que, a medida que se avanza en la edad el IMC, guarda relación con los niveles de rendimiento motor, siendo mejor en los normales comparados con los de sobrepeso. Lopes et al. (2014) plantean cómo los niños con mayor cociente motor demostraron niveles más bajos de IMC y las diferencias entre estas variables aumentaron a lo largo de la edad.

Otros estudios muestran cómo la coordinación motriz moderada se asocia negativamente con el IMC y la asociación aumenta durante la infancia $(-0,16$ a $-0,50)$. Los niños con sobrepeso y con obesidad, de ambos sexos, mostraron nive-

Tabla 1. Estadística (media, D.S, IC, significancia) de la coordinación motriz, según la ciudad, el tipo de colegio, el sexo y la edad de los participantes.

\begin{tabular}{|c|c|c|c|c|}
\hline \multirow{2}{*}{ Variables } & \multicolumn{2}{|c|}{ Coordinación motriz } & \multicolumn{2}{|c|}{ Estadístico de prueba } \\
\hline & Media & D.S & Kruskal Wallis & Sig. (bilateral) \\
\hline Ciudad & & & \multirow{7}{*}{990,923} & \multirow{7}{*}{$0,000 *$} \\
\hline Manizales & 201,67 & 34,677 & & \\
\hline Riohacha & 194,5 & 30,496 & & \\
\hline Popayán & 134,8 & 30,146 & & \\
\hline Buga & 197,04 & 33,941 & & \\
\hline Zarzal & 166,2 & 23,636 & & \\
\hline Yarumal & 190,2 & 23,355 & & \\
\hline Tipo de Colegio & & & U de Mann Whitney & Sig. (bilateral) \\
\hline Publico & 25151 & 2,677 & \multirow{2}{*}{25151} & \multirow{2}{*}{$0,002 *$} \\
\hline Privado & 180,6 & 1,337 & & \\
\hline Sexo & & & U de Mann Whitney & Sig. (bilateral) \\
\hline Hombres & 189,3 & 38,846 & \multirow{2}{*}{64554,5} & \multirow{2}{*}{$0,000^{*}$} \\
\hline Mujeres & 172,4 & 34,939 & & \\
\hline Edad & & & Kruskal Wallis & Sig. (bilateral) \\
\hline 10 años & 174,4 & 37,230 & \multirow{3}{*}{23,775} & \multirow{3}{*}{$0,000 *$} \\
\hline 11 años & 181,2 & 37,086 & & \\
\hline 12 años & 187,5 & 38,2187 & & \\
\hline
\end{tabular}

D.S. Desviación Estándar; *p<0,05.

Tabla 2. Diferencia de medias de Índice de Masa Corporal por sexo y por edad.

\begin{tabular}{|c|c|c|c|c|c|c|}
\hline \multirow{2}{*}{ Edad } & \multirow{2}{*}{ Sexo } & \multirow{2}{*}{$\mathrm{n}$} & \multirow{2}{*}{ Media } & \multirow{2}{*}{$\begin{array}{l}\text { Desviación } \\
\text { estándar }\end{array}$} & \multicolumn{2}{|c|}{ Estadístico de Prueba } \\
\hline & & & & & U de Mann-Whitney & Sig. (bilateral) \\
\hline \multirow{2}{*}{10 años } & Hombre & 461 & 18,18 & 3,1966 & \multirow{2}{*}{113160,000} & \multirow{2}{*}{0,744} \\
\hline & Mujer & 497 & 18,06 & 3,0124 & & \\
\hline \multirow{2}{*}{11 años } & Hombre & 430 & 18,85 & 3,2476 & \multirow{2}{*}{91998,000} & \multirow{2}{*}{0,596} \\
\hline & Mujer & 437 & 18,89 & 3,0114 & & \\
\hline \multirow{2}{*}{12 años } & Hombre & 417 & 18,93 & 2,9900 & \multirow{2}{*}{75662,000} & \multirow{2}{*}{$0,005^{*}$} \\
\hline & Mujer & 409 & 19,41 & 2,9034 & & \\
\hline
\end{tabular}

$* p<0,05$. 
Tabla 3. Estadística descriptiva (media, D.S, IC) del IMC, según la ciudad, el tipo de colegio, el sexo y la edad de los participantes.

\begin{tabular}{|c|c|c|c|c|}
\hline \multirow{2}{*}{ Variables } & \multirow{2}{*}{ Media } & \multirow{2}{*}{ D.S. } & \multicolumn{2}{|c|}{ Estadístico de prueba } \\
\hline & & & Kruskal Wallis & Sig. (bilateral) \\
\hline Ciudad & Índice de & asa corporal & \multirow{7}{*}{$4571,785^{\mathrm{a}}$} & \multirow{7}{*}{$0,000 *$} \\
\hline Manizales & 18,177 & 2,82 & & \\
\hline Riohacha & 18,078 & 2,92 & & \\
\hline Popayán & 18,860 & 2,79 & & \\
\hline Buga & 18,951 & 3,28 & & \\
\hline Zarzal & 19,401 & 3,748 & & \\
\hline \multirow[t]{2}{*}{ Yarumal } & 18,704 & 2,694 & & \\
\hline & \multicolumn{2}{|c|}{ Tipo de Colegio } & U de Mann Whitney & Sig. (bilateral) \\
\hline Publico & 18,640 & 3,177 & \multirow{2}{*}{878,44} & \multirow{2}{*}{$0,000 *$} \\
\hline \multirow[t]{2}{*}{ Privado } & 18,809 & 2,914 & & \\
\hline & & xo & U de Mann Whitney & Sig. (bilateral) \\
\hline Hombres & 18,642 & 3,165 & \multirow{2}{*}{942,478} & \multirow{2}{*}{0,011} \\
\hline Mujeres & 18,744 & 3,029 & & \\
\hline Edad & & & Kruskal Wallis & Sig. (bilateral) \\
\hline 10 años & 18,120 & 3,10 & \multirow{3}{*}{1993,711} & \multirow{3}{*}{$0,000 *$} \\
\hline 11 años & 18,874 & 3,12 & & \\
\hline 12 años & 19,169 & 2,95 & & \\
\hline
\end{tabular}

D.S Estándar; Desviación $S^{*} p<0,05$.

les más bajos de coordinación motriz que los niños de peso normal (Melo \& Lopes, 2013). Robinson et al. (2015), Lopes et al. (2012) y Lopes et al. (2011) evidencian, además, cómo los buenos niveles de coordinación motriz pueden estar asociados a valores más altos de AF y ser así, un factor preventivo del desarrollo de exceso de peso.

De los 2.651 niños evaluados en el presente estudio, la media de la edad fue de 10,95 años \pm 0,819 años; los hombres presentan una mejor coordinación que las mujeres y, a medida que se avanza en la edad, se mejora la coordinación motriz (Melo \& López, 2013), siendo ésta una correlación inversa, es decir, a menor IMC mayores niveles de coordinación, resultados muy similares a los encontrados por D'Hondt et al. (2014) y D'Hondt et al. (2011).

Bustamante (2008), en su estudio sobre la coordinación motora y la influencia de la edad y la adiposidad señala, también, que los niños/niñas con niveles de adiposidad alto muestran disminución en los parámetros de las pruebas motoras, aplicadas a través del test KTK; los niños peruanos, para el caso del este estudio, presentaron niveles de adiposidad elevada y media y, de igual manera, se refleja los niveles bajos en el desarrollo de las habilidades coordinativas. Además, muestra este estudio, que los niveles de coordinación también me- joran con la edad, donde por cada año que aumenta, incrementa 2 saltos, confirmando la hipótesis de la maduración biológica, que va dando la edad para la coordinación motriz.

En cuanto a la coordinación y el sexo, se encontró en el presente estudio, que las diferencias de las medias es mayor en los hombres y es estadísticamente significativa $(p>0,000)$, es decir, a medida que aumenta la edad mejoran los niveles de coordinación; en este mismo sentido, Santos (2013), en su estudio, observó diferencias en la coordinación motora relacionadas con el género y con el IMC (sobrepeso/obesidad versus normopeso), lo que les permitió concluir, que los niños y las niñas con normopeso tienen una coordinación motora significativamente superior que los niños con sobrepeso/obesidad $(\mathrm{p} \leq 0,02)$, información que coincide con otra investigación (Araújo Barroso et al. 2011), donde el nivel de adiposidad presenta una influencia negativa en cada prueba de coordinación y también en la coordinación total.

El coeficiente de correlación de Pearson muestra correlaciones significativas e inversas, entre la coordinación motriz y el IMC, en todos los grupos de las variables estudiadas. Se destaca, que las correlaciones son más altas en ciudades, como Zarzal y Yarumal, en los niños de colegios oficiales, en los hombres y en los de mayor edad (Tabla 4). 
Tabla 4. Correlación entre IMC vs Nivel de coordinación por ciudad, por tipo de colegio, por sexo y por edad.

\begin{tabular}{|l|c|c|}
\hline \multicolumn{1}{|c|}{ Ciudad } & Coeficiente de correlación & Sig. (bilateral) \\
\hline Manizales & $-0,127$ & $0,000^{*}$ \\
\hline Riohacha & $-0,226$ & $0,000^{*}$ \\
\hline Popayán & $-0,194$ & $0,000^{*}$ \\
\hline Zarzal & $-0,232$ & $0,000^{*}$ \\
\hline Yarumal & $-0,255$ & $0,000^{*}$ \\
\hline Tipo de colegio \\
\hline Público & $-0,217$ & $0,000^{*}$ \\
\hline Privado & $-0,167$ & $0,000^{*}$ \\
\hline Sexo & $0,000^{*}$ \\
\hline Hombres & 0,209 & $0,000^{*}$ \\
\hline Mujeres & -0141 & $0,00 *^{*}$ \\
\hline Edad & $0,00 *^{*}$ \\
\hline 10 años & $-0,189$ & $0,000^{*}$ \\
\hline 11 años & $-0,193$ & \\
\hline 12 años & $-0,298$ & \\
* p <0,05. &
\end{tabular}

En el presente estudio, se encontró, también, que existen diferencias entre los niveles de coordinación de los niños con las diferentes variables abordadas por sexo, donde los hombres presentan una mejor coordinación que las mujeres y a mayor edad mejores niveles de coordinación, siendo estas diferencias, estadísticamente significativas. Al comparar estos datos con otros estudios, se evidencia, como resultados similares, una diferencia significativa a favor de los hombres en comparación con las mujeres (Lopes et al. 2003; Torralba et al. 2014).

Por otro lado, diversos estudios revelan que son más activos los niños que las niñas, donde calculan los minutos que dedican a la práctica de la actividad física, durante toda la semana, encontrando que el tiempo dedicado por los hombres resultó significativamente superior al dedicado por las mujeres (Monti et al. 2005; Luengo Vaquero, 2007; Bustamante, 2008; Valdivia et al. 2008).

Como conclusión, los niveles de coordinación motriz se mejoran en la medida que se avanza con la edad, siendo mejores los desempeños para los hombres y la existencia de correlaciones inversas entre el IMC y las diferentes variables del estudio, siendo estas correlaciones mayores en los niños de mayor edad, hombres y en los colegios oficiales.

Agradecimientos: Los autores expresan sus agradecimientos a la Universidad Autónoma de Manizales y a los niños e instituciones educativas, quienes participaron en el estudio.
Conflicto de intereses: Los autores declaran que no existen conflictos de intereses en relación con la investigación presentada.

\section{BIBLIOGRAFÍA}

1. ARAÚJO BARROSO G., M. de C.; MOURÃO, I. 2011. A Influência da Obesidade na Coordenação Motora em crianças do 1o Ciclo do Ensino Básico. Universidade de Trás-os-Montes e Alto Douro. 66p.

2. BUSTAMANTE, V. 2008. Coordinación Motora: Influencia de la edad, sexo, estatus socio-económico y niveles de adiposidad en niños peruanos. Rev. Bras. Cineantropometria e Desempenho Humano. 10(1):25-34.

3. CHIVERS, P.; LARKIN, D.; ROSE, E.; BEILIN, L.; HANDS, B. 2013. Low motor performance scores among overweight children: poor coordination or morphological constraints? Hum Mov Sci. 32(5):1127-37.

4. D'HONDT, E.; DEFORCHE, B.; GENTIER, I.; VERSTUYF, J.; VAEYENS, R.; DE BOURDEAUDHUIJ, I.; RENNAT., M.L. 2014. A longitudinal study of gross motor coordination and weight status in children. Obesity (Silver Spring). 22(6):1505-1511.

5. D'HONDT, E.; DEFORCHE, B.; VAEYENS, R.; VANDORPE, B.; VANDENDRIESSCHE, J.; PION, J.; PION, 
J.; PHILIPPAERST, R.; DE BOURDEAUDHUJ, I.; LENOIR, M. 2011. Gross motor coordination in relation to weight status and age in 5- to 12-year-old boys and girls: a cross-sectional study. Int JPediatr Obes. 6(2-2):e556-64.

6. DUQUE, I.; PARRA, J. 2012. Exposición a pantallas, sobrepeso y desacondicionamiento físico en niños y niñas. Rev. Latinoam. Ciencias Sociales, Niñez y Juventud. 10(2):971-981.

7. FERNÁNDEZ, M. 2005. Manejo práctico del niño obeso y con sobrepeso en pediatría de atención primaria. Rev. Foro Pediátrico. 2(supl. 1):61-69.

8. GALLAHUE, D.L.; OZMUN, J.C. 2005. Compreendendo o desenvolvimento motor: bebês, crianças, adolescentes e adultos. São Paulo: Phorte Editora. 585p.

9. GRAHAM, D.J.; SIRARD, J.R; NEUMARK-SZTAINER, D. 2011. Adolescents' attitudes toward sports, exercise, and fitness predict physical activity 5 and 10 years later. Prev. Med. 52(2):130-132.

10. HERNÁNDEZ, J.; VELÁZQUIEZ, R.; ALONSO, D. 2004. La evaluación en educación física: investigación y práctica en el ámbito escolar. Barcelona: Graó Editorial. 200p.

11. LOPES, L.; SANTOS, R.; PEREIRA, B.; LOPES, V. 2013. Associations between gross motor coordination and academic achievement in elementary school children. Hum. Mov. Sci. 32(1):9-20.

12. LOPES, V.; STODDEN, D.; BIANCHI, M.; MAIA, J.; RODRIGUES, L. 2012. Correlation between BMI and motor coordination in children. J. Sci. Med. Sport. 15(1):38-43.

13. LOPES, V.P.; STODDEN, D.F.; RODRIGUES, L.P. 2014. Weight status is associated with cross-sectional trajectories of motor co-ordination across childhood. Child: Care, Health and Development. 40(6):891899.

14. LOPES, V.; MAIA, J.; SILVA, R.; SEABRA, A.; MORAIS, F. 2003. Estudo do nível de desenvolvimento da coordenação motora da população escolar (6 a 10 años de idade) da Região Autónoma dos Açores. Rev. Portuguesa de Ciências do Desporto. 3(1):47-60.

15. LOPES, V.P.; MAIA, J.A.R.; RODRIGUES, L.P.; MALINA, R.M. 2012. Motor coordination, physical activity and fitness as predictors of longitudinal change in adi- posity during childhood. European J. Sport Science. 12(4):384-391.

16. LOPES, V.P.; MAIA, J.A.R.; RODRIGUES, L.P.; MALINA, R.M. 2011. Motor coordination as predictor of physical activity in childhood. Scandinavian J. Medicine \& Science in Sports. 21(5):663-669.

17. KIPHARD, E.; SCHILLING, F. 1974. The KörperkoordinationsTest für Kinder. KTK Manual. Weiheim: Beltz Test GmbH.

18. LUENGO VAQUERO, C. 2007. Actividad físico-deportiva extraescolar en alumnos de primaria. Rev. Internacional de Medicina y Ciencias de la Actividad Física y el Deporte. 7(27):174-184.

19. MELO, M.; LOPES, V. 2013. Associacao entre o indice de massa corporal e a coordenacao motora em criancas. Rev Bras Educ Fis Esporte. 27(1):7-13.

20. MONTI, M.; BARRIOPEDRO, M.; OLIVÁN, J. 2005. El sedentarismo en la infancia. Los niveles de actividad física en niños/as de la Comunidad Autónoma de Madrid. Apunts, Educación Física y Deportes. 82:511.

21. MUÑOZ RIVERA, D. 2009. La coordinación y el equilibrio en el área de Educación Física. Actividades para su desarrollo. Rev. digital EFdeportes. 13(130):11p. Disponible desde Internet en: http://www.efdeportes. com (con acceso 15/04/2013).

22. ORGANIZACIÓN PANAMERICANA DE LA SALUD. Oficina Regional de la Organización Mundial de la Salud (OPS/OMS). 2003. Instituto Nacional de Salud, Salud y Desplazamiento en cuatro ciudades: Cali, Cartagena, Montería y Soacha, 2002-2003. Los informes técnicos de estas investigaciones están disponibles en Internet en: www.disaster-info.net/desplazados/_con acceso 28/02/2015).

23. RAMOS, S. 2006. Prevalencia de sobrepeso y obesidad en escolares de secundaria en colegios privados de la ciudad de Manizales. Disponible desde Internet en: www.reddeporte.com/ARTICULOS/0002/ARTICULO\%20CIENTIFICO\%20sobrepeso\%20obesidad\%5B1\%5Dsanctiago.htm (con acceso 26/02/2014).

24. ROBINSON, L.E.; STODDEN, D.F.; BARNETT, L.M.; LOPES, V.P.; LOGAN, S.W.; RODRIGUES, L.P.; D'HONDT, E. 2015. Motor competence and its effect on positive developmental trajectories of health. Sports Medicine. 45(9):1273-1284. 
25. RUIZ PÉREZ, L.M. 2005. Moverse con dificultad en la escuela. Introducción a los problemas evolutivos de coordinación motriz. Sevilla: Wanceulen. Ed. Deportiva. 192p.

26. SANTOS, L.B. 2013. Desarrollo de las habilidades motoras fundamentales en función del sexo y del índice de masa corporal en escolares. Cuad. Psicol. del Deport. 13:63-71.

27. SECIEN-PALACIN, J.; JACOBY, E. 2003. Sociodemographic and enviromental factors associated with sports physical activity in the urban population of Peru. Rev. Panam. Salud Publica. 14(4):255-264.

28. TORRALBA, M.; VIEIRA, M.; LLEIXÀ, T.; GORLA, J. 2014. Evaluación de la Coordinación Motora en Educación Primaria de Barcelona y Provincia. Rev. Int. Med. y Ciencias la Act. Física y el Deporte. 16(62):355-371.

29. VALDIVIA, A.; CARTAGENA, L.; SARRIA, N.; TÁVARA, I.; SEABRA, A.; SILVA, R. 2008. Coordinación motora: influencia de la edad, sexo, estatus socioeconómico y niveles de adiposidad en niños peruanos. Rev. Bras. Cineantropometria y Desempenho Humano. 10(1):25-34.

30. VANDORPE, B.; SCHILLING, F. 2011. The Körperkoordinations Test für Kinder: reference values and suitability for 6-12-year-old children in Flanders. Scandinavian J. Medicine \& Science in Sports. 21:378-388.

31. VIDAL, S.; BUSTAMANTE, A.; LOPES, V.; SEABRA, A.; SILVA, R.; MAIA J. 2009. Construção de cartas centílicas da coordenação motora de crianças dos 6 aos 11 años da Região Autónoma dos Açores, Portugal. Rev. Portuguesa de Ciência do Desporto. 9(1):24-35.

Recibido: Noviembre 13 de 2017

Aceptado: Abril 11 de 2018

\section{Cómo citar:}

Vidarte-Claros, J.A.; Vélez Álvarez, C.; Parra-Sánchez, J.H. 2018. Coordinación motriz e índice de masa corporal en escolares de seis ciudades colombianas. Rev. U.D.C.A. Act.\& Div. Cient. 21(1): 15-22. 